\title{
ABOUT ONE APPROACH TO NUMERICAL SOLUTION OF NONLINEAR OPTIMAL SPEED PROBLEMS
}

\author{
A.S. Buldaev ${ }^{1}$, I.D. Burlakov ${ }^{1}$ \\ ${ }^{1}$ Buryat State University, Ulan-Ude, Russian Federation \\ E-mails: buldaev@mail.ru, ivan.burlakov.91@mail.ru
}

\begin{abstract}
Optimal speed problems are among the most important problems of the theory of controlled systems. In the qualitative theory of nonlinear speed problems one of the main results is the Pontryagin maximum principle. For the numerical solution of nonlinear speed problems, along with methods based on the maximum principle, methods of reducing to auxiliary problems of optimal control using linearization, parameterization, discretization, and other techniques are widely used. The complexity of numerical methods is determined by the number of iterations to find a solution to the speed problem with a given accuracy. A universal computational procedure that is effective for calculating a variety of speed problems does not currently exist. Therefore, it is actual to develop special approaches to reduce the amount of calculations and reduce the number of iterations. The paper proposes a new approach based on the reduction of a nonlinear speed problem to an auxiliary optimization problem with mixed control functions and parameters. To search for a solution to the emerging auxiliary problem, a specially developed form of conditions for nonlocal improvement of admissible control in the form of a fixed-point problem of the control operator, and a constructed iterative algorithm for successive improvement of admissible controls are used. Approbation and comparative analysis of the computational efficiency of the proposed fixed point approach is carried out on known models of optimal speed problems.
\end{abstract}

Keywords: optimal speed problem; conditions for improving control; fixed point problem.

\section{Introduction}

A common approach $[1,2]$ to the numerical solution of optimal speed problems is reduction by the method of penalty functionals to an auxiliary problem with a free right end and an unfixed control time. The arising auxiliary problem by changing the time variable can be transformed to the optimization problem for control functions and parameters with a fixed time entering the following class of problem:

$$
\begin{gathered}
\Phi(\sigma)=\varphi\left(x\left(t_{1}\right), \omega\right)+\int_{T} F(x(t), u(t), \omega, t) d t \rightarrow \inf _{\sigma \in \Omega}, \\
\dot{x}(t)=f(x(t), u(t), \omega, t), \quad x\left(t_{0}\right)=a, \\
u(t) \in U, \omega \in W, a \in A, \quad t \in T=\left[t_{0}, t_{1}\right],
\end{gathered}
$$

in which $x(t)=\left(x_{1}(t), \ldots, x_{n}(t)\right)$ is a state vector, $u(t)=\left(u_{1}(t), \ldots, u_{m}(t)\right)$ is a vector of control functions, $\omega=\left(\omega_{1}, \ldots, \omega_{l}\right)$ and $a=\left(a_{1}, \ldots, a_{n}\right)$ are vectors of control parameters. Sets $U \subseteq R^{m}, W \subseteq R^{l}, A \subseteq R^{n}$ are compact and convex. Interval $T$ is fixed. As admissible control functions, we consider a set $V$ of piecewise-continuous functions on $T$ with values in set $U, \sigma=(u, \omega, a)$ is admissible control with values in set $\Omega=V \times W \times A$. Function $\varphi(x, \omega)$ is continuously differentiable on $R^{n} \times W$, functions $F(x, u, \omega, t), f(x, u, \omega, t)$ and their partial derivatives with respect to $x, u, \omega$ are continuous in the set of arguments on set 
$R^{n} \times U \times W \times T$. Function $f(x, u, \omega, t)$ satisfies the Lipschitz condition to $x$ in $R^{n} \times U \times W \times T$ with a constant $L>0:\|f(x, u, \omega, t)-f(y, u, \omega, t)\| \leq L\|x-y\|$. Conditions guarantee the existence and uniqueness of the solution $x(t, \sigma), t \in T$ of system (2) for any admissible control $\sigma \in \Omega$.

In [4-7] special methods for sequential improvement of control and search for extreme controls were developed in the class of problems (1), (2) and other classes, based on the solution, respectively, of the conditions for non-local control improvement and the necessary optimality conditions in the form of the fixed point problems defined control operators. These methods are the development and generalization of non-local methods for optimizing controls based on non-standard approximations of functionals of problems without residual terms of expansions in linear and linearly quadratic in the problems of optimal control [3].

In this paper, the fixed-point approach proposed in [4-7] is concretized and tested in comparison with the known methods for computational efficiency with reference to known model problems of optimal speed.

\section{Conditions for Improving Control}

The problem of improving control in the class of problems (1), (2) is considered in the following general formulation: for a given control $\sigma^{I} \in \Omega$ need to find control $\sigma \in \Omega$ with the condition $\Delta_{\sigma} \Phi\left(\sigma^{I}\right)=\Phi(\sigma)-\Phi\left(\sigma^{I}\right) \leq 0$.

Pontryagin function $H$ with conjugate variable $\psi \in R^{n}$ and the standard conjugate system have the form

$$
\begin{gathered}
H(\psi, x, u, \omega, t)=\langle\psi, f(x, u, \omega, t)\rangle-F(x, u, \omega, t) \\
\dot{\psi}(t)=-H_{x}(\psi(t), x(t), u(t), \omega, t), \quad t \in T, \quad \psi\left(t_{1}\right)=-\varphi_{x}\left(x\left(t_{1}\right), \omega\right) .
\end{gathered}
$$

For an admissible control $\sigma \in \Omega$ we denote $\psi(t, \sigma), t \in T$ - the solution of the standard conjugate system (3) for $x(t)=x(t, \sigma)$ and arguments $u, \omega$, corresponding to the control components $\sigma$. A special increment of an arbitrary vector-valued function $g\left(y_{1}, \ldots, y_{l}\right)$ with respect to $y_{s_{1}}, y_{s_{2}}$ will be denoted by

$$
\begin{gathered}
\Delta_{y_{s_{1}}+\Delta y_{s_{1}}, y_{s_{2}}+\Delta y_{s_{2}}} g\left(y_{1}, \ldots, y_{l}\right)= \\
=g\left(y_{1}, \ldots, y_{s_{1}}+\Delta y_{s_{1}}, \ldots, y_{s_{2}}+\Delta y_{s_{2}}, \ldots, y_{l}\right)-g\left(y_{1}, \ldots, y_{l}\right) .
\end{gathered}
$$

In addition, we denote $\Delta x(t)=x(t, \sigma)-x\left(t, \sigma^{I}\right), \Delta u(t)=u(t)-u^{I}(t), \Delta \omega=\omega-\omega^{I}$, $\Delta a=a-a^{I}$.

We introduce a modified differential-algebraic conjugate system including an additional phase variable $y(t)=\left(y_{1}(t), \ldots, y_{n}(t)\right)$ in the form

$$
\begin{gathered}
\dot{p}(t)=-H_{x}(p(t), x(t), u(t), \omega, t)-r(t), \\
\left\langle H_{x}(p(t), x(t), u(t), \omega, t)+r(t), y(t)-x(t)\right\rangle= \\
\Delta_{y(t)} H(p(t), x(t), u(t), \omega, t)
\end{gathered}
$$

with boundary conditions

$$
\begin{gathered}
p\left(t_{1}\right)=-\varphi_{x}\left(x\left(t_{1}\right), \omega\right)-q \\
\left\langle\varphi_{x}\left(x\left(t_{1}\right), \omega\right)+q, y\left(t_{1}\right)-x\left(t_{1}\right)\right\rangle=\Delta_{y\left(t_{1}\right)} \varphi\left(x\left(t_{1}\right), \omega\right),
\end{gathered}
$$


in which by definition we set $r(t)=0, q=0$ in the case of the linearity of the functions $\varphi, F, f$ with respect $x$ (the linear problem in the state (1), (2)), and also in the case of $y(t)=x(t)$ with the corresponding $t \in T$.

In the linear problem in the state (1), (2), the modified conjugate system (4) - (7), by definition, coincides with the standard conjugate system (3).

In the nonlinear problem (1), (2), the algebraic equations (5) and (7) can always be analytically resolved with respect to the quantities $r(t)$ and $q$ in the form of explicit or conditional formulas (perhaps not uniquely).

The universal method of resolution is the following rule (using equation (5) as an example). If there exists $k \in\{1, \ldots, n\}$, for which $y_{k}(t) \neq x_{k}(t)$, then for $i \in\{1, \ldots, n\}$ we set

$$
\begin{gathered}
r_{i}(t)=0, i \neq k \\
r_{i}(t)=\frac{\Delta_{y(t)} H(p(t), x(t), u(t), \omega, t) d t}{y_{i}(t)-x_{i}(t)}-\frac{\left\langle H_{x}(p(t), x(t), u(t), \omega, t), y(t)-x(t)\right\rangle}{y_{i}(t)-x_{i}(t)}, i=k .
\end{gathered}
$$

If for all $k \in\{1, \ldots, n\}$ we have $y_{k}(t)=x_{k}(t)$, then by definition $r(t)=0$.

An alternative simple method of explicit resolution can be applied to the polynomial problem (1), (2) (functions $\varphi, F, f$ are polynomial in the variable $x$ ), using the Taylor formula for the polynomial. In particular, in the problem (1), (2) quadratic in the state, we obtain (using the example of equation (5))

$$
r(t)=\frac{1}{2} H_{x x}(p(t), x(t), u(t), \omega, t)(y(t)-x(t)) .
$$

Thus, the differential-algebraic conjugate system $(4)-(7)$ can always be reduced (possibly not uniquely) to a differential conjugate system with uniquely determined quantities $r(t)$ and $q$.

For admissible controls $\sigma \in \Omega, \sigma^{I} \in \Omega$ denote $p\left(t, \sigma^{I}, \sigma\right), t \in T$ - solution of the modified conjugate system $(4)-(7)$ for $x(t)=x\left(t, \sigma^{I}\right), y(t)=x(t, \sigma), u(t)=u^{I}(t)$, $\omega=\omega^{I}$. The definition implies the obvious equality $p(t, \sigma, \sigma)=\psi(t, \sigma), t \in T$.

In [6], in the class of problems (1), (2) with the help of the considered modification of the standard conjugate system, a non-standard formula for the increment of the functional that does not contain the remainder terms of the expansions

$$
\begin{gathered}
\Delta_{\sigma} \Phi\left(\sigma^{I}\right)=-\Delta_{\omega}\left\{-\varphi\left(x\left(t_{1}, \sigma\right), \omega^{I}\right)+\int_{T} H\left(p\left(t, \sigma^{I}, \sigma\right), x(t, \sigma), u(t), \omega^{I}, t\right) d t\right\}- \\
-\left\langle p\left(t_{0}, \sigma^{I}, \sigma\right), \Delta a\right\rangle-\int_{T} \Delta_{u(t)} H\left(p\left(t, \sigma^{I}, \sigma\right), x(t, \sigma), u^{I}(t), \omega^{I}, t\right) d t .
\end{gathered}
$$

We denote by $P_{Y}$ the projection operator onto set $Y \subset R^{k}$ in the Euclidean norm

$$
P_{Y}(z)=\arg \min _{y \in Y}(\|y-z\|), z \in R^{k} .
$$

For a given $\sigma^{I} \in \Omega$ consider the following system of equations for the control $\sigma=(u, \omega, a)$ with the projection parameter $\alpha>0$ :

$$
\begin{gathered}
u(t)=P_{U}\left(u^{I}(t)+\alpha\left(H_{u}\left(p\left(t, \sigma^{I}, \sigma\right), x(t, \sigma), u^{I}(t), \omega^{I}, t\right)+s^{u}(t)\right)\right), \quad t \in T, \\
\Delta_{u(t)} H\left(p\left(t, \sigma^{I}, \sigma\right), x(t, \sigma), u^{I}(t), \omega^{I}, t\right)= \\
=\left\langle H_{u}\left(p\left(t, \sigma^{I}, \sigma\right), x(t, \sigma), u^{I}(t), \omega^{I}, t\right)+s^{u}(t), u(t)-u^{I}(t)\right\rangle, \\
\omega=P_{W}\left(\omega^{I}+\alpha\left(-\varphi_{\omega}\left(x\left(t_{1}, \sigma\right), \omega^{I}\right)+\int_{T} H_{\omega}\left(p\left(t, \sigma^{I}, \sigma\right), x(t, \sigma), u(t), \omega^{I}, t\right) d t+s^{\omega}\right)\right),
\end{gathered}
$$




$$
\begin{gathered}
\Delta_{\omega}\left\{-\varphi\left(x\left(t_{1}, \sigma\right), \omega^{I}\right)+\int_{T} H\left(p\left(t, \sigma^{I}, \sigma\right), x(t, \sigma), u(t), \omega^{I}, t\right) d t\right\}= \\
=\left\langle-\varphi_{\omega}\left(x\left(t_{1}, \sigma\right), \omega^{I}\right)+\int_{T} H_{\omega}\left(p\left(t, \sigma^{I}, \sigma\right), x(t, \sigma), u(t), \omega^{I}, t\right) d t+s^{\omega}, \omega-\omega^{I}\right\rangle, \\
a=P_{A}\left(a^{I}+\alpha p\left(t_{0}, \sigma^{I}, \sigma\right)\right),
\end{gathered}
$$

in which, by definition, in (10) $s^{u}(t)=0$ in the case of the linearity of functions $F, f$ with respect to $u$ (problem (1), (2) linear in the control function), or in case $u(t)=u^{I}(t)$ at $t \in T$. Similarly, in (12), by definition, $s^{\omega}=0$ is assumed in the case of linearity of functions $F, f$ with respect to $\omega$ (problem (1), (2) linear in the parameter $\omega$ ), as well as for $\omega=\omega^{I}$.

In system (9) - (13), which is bilinear in control of problem (1), (2) (function $F, f$ are linear in each variable $u$ and $\omega$ ) does not contain equations (10) and (12). In other problems that are non-linear in control function $u$ and control parameter $\omega$ in problem (1), (2), the corresponding equations (10) and (12) can always be uniquely resolved with respect to quantities $s^{u}(t)$ and $s^{\omega}$. In particular, similarly to the above rules for equation (5) (perhaps not in a unique way).

It was shown in [7] that admissible solution $\sigma=(u, \omega, a)$ of system (9) - (13) provides an improvement in the control $\sigma^{I} \in \Omega$ for any parameter $\alpha>0$ with an estimate of the improvement of the functional:

$$
\Delta_{\sigma} \Phi\left(\sigma^{I}\right) \leq-\frac{1}{\alpha} \int_{T}\left\|u(t)-u^{I}(t)\right\|^{2} d t-\frac{1}{\alpha}\left\|\omega-\omega^{I}\right\|^{2}-\frac{1}{\alpha}\left\|a-a^{I}\right\|^{2} .
$$

At the same time, control improvement is guaranteed not only in a fairly small neighbourhood of initial control $\sigma^{I} \in \Omega$, that is, the improvement procedure under consideration has a nonlocality property, unlike known gradient methods and other local methods for improving control.

It is proposed to consider conditions (9) - (13) as a fixed point problem in control space for the uniquely chosen control operator defined by the right-hand sides of these conditions. This allows us to apply and modify the developed fixed point theory and methods for realizing the conditions for improving control and constructing iterative methods for solving optimal control problems. The developed approach of fixed points consists of the sequential solution of problems of improving control in the form of the constructed problems on the fixed point (9) - (13) of a uniquely determined control operator.

\section{Zermelo Problem}

In [2], the known optimal speed problem (Zermelo problem) is reduced by the penalty method to the following optimal control problem with non-fixed control time

$$
\begin{aligned}
& \begin{cases}\dot{x}_{1}(t)=\cos x_{3}(t), & x_{1}(0)=0, \\
\dot{x}_{2}(t)=\sin x_{3}(t), & x_{2}(0)=0, \\
\dot{x}_{3}(t)=u(t), & x_{3}(0)=0\end{cases} \\
& t \in\left[0, t_{1}\right], \quad|u(t)| \leq 0,5 . \\
& G\left(u, t_{1}\right)=t_{1}+1000\left(\left(x_{1}\left(t_{1}\right)-4\right)^{2}+\left(x_{2}\left(t_{1}\right)-3\right)^{2}\right) \rightarrow \min .
\end{aligned}
$$

In the problem, we can assume that quantity $t_{1}$ is bounded: $t_{1} \leq \bar{t}, \bar{t}>0$. 
Making the replacement of time by rule

$$
\begin{gathered}
t(\tau)=t_{0}+\left(t_{1}-t_{0}\right) \tau=\omega \tau, \quad t_{0}=0, \quad \tau \in[0,1], \quad \omega=t_{1} \geq 0 \\
v(\tau)=u(t(\tau)), \quad y(\tau)=x(t(\tau)), \quad \sigma=(v, \omega),
\end{gathered}
$$

this problem reduces to the problem with a fixed time:

$$
\begin{aligned}
& \begin{cases}\dot{y}_{1}(\tau)=\omega \cos y_{3}(\tau), & y_{1}(0)=0 \\
\dot{y}_{2}(\tau)=\omega \sin y_{3}(\tau), & y_{2}(0)=0 \\
\dot{y}_{3}(\tau)=\omega v(\tau), & y_{3}(0)=0\end{cases} \\
& \Phi(\sigma)=\omega+1000\left(\left(y_{1}(1)-4\right)^{2}+\left(y_{2}(1)-3\right)^{2}\right) \rightarrow \min , \\
& T=[0,1], \quad U=\{u:|u| \leq 0,5\}, \quad W=\{\omega: 0 \leq \omega \leq \bar{t}\} .
\end{aligned}
$$

The obtained auxiliary problem with fixed time is bilinear with respect to controllable variables $v$ and $\omega$, which greatly simplifies the structure of the fixed point problem for control improvement. The Pontryagin function and modified differential-algebraic conjugate system $(4)-(7)$ take the following form:

$$
\begin{aligned}
& H(p, y, v, \omega, \tau)=\omega\left(p_{1} \cos y_{3}+p_{2} \sin y_{3}+p_{3} v\right), \\
& \left\{\begin{array}{l}
\dot{p}_{1}(\tau)=-r_{1}(\tau), \\
\dot{p}_{2}(\tau)=-r_{2}(\tau), \\
\dot{p}_{3}(\tau)=\omega p_{1}(\tau) \sin y_{3}(\tau)-\omega p_{2}(\tau) \cos y_{3}(\tau)-r_{3}(\tau),
\end{array}\right. \\
& \left\{\begin{array}{l}
p_{1}(1)=-2000\left(y_{1}(1)-4\right)-q_{1}, \\
p_{2}(1)=-2000\left(y_{2}(1)-3\right)-q_{2} \\
p_{3}(1)=-q_{3} .
\end{array}\right.
\end{aligned}
$$

In this case, the variable $r(\tau)=\left(r_{1}(\tau), r_{2}(\tau), r_{3}(\tau)\right)$ is determined from an algebraic equation with an additional phase variable, for which notation $z(t)=\left(z_{1}(t), z_{2}(t), z_{3}(t)\right)$ is used:

$$
\begin{gathered}
\omega p_{1}(\tau)\left(\cos z_{3}(\tau)-\cos y_{3}(\tau)\right)+\omega p_{2}(\tau)\left(\sin z_{3}(\tau)-\sin y_{3}(\tau)\right)= \\
=r_{1}(\tau)\left(z_{1}(\tau)-y_{1}(\tau)\right)+r_{2}(\tau)\left(z_{2}(\tau)-y_{2}(\tau)\right)+ \\
+\left(-\omega p_{1}(\tau) \sin y_{3}(\tau)+\omega p_{2}(\tau) \cos y_{3}(\tau)+r_{3}(\tau)\right)\left(z_{3}(\tau)-y_{3}(\tau)\right)
\end{gathered}
$$

The value of $q=\left(q_{1}, q_{2}, q_{3}\right)$ is determined from equation:

$$
\begin{gathered}
1000\left(\left(z_{1}(1)-4\right)^{2}-\left(y_{1}(1)-4\right)^{2}+\left(z_{2}(1)-3\right)^{2}-\left(y_{2}(1)-3\right)^{2}\right)= \\
=\left(2000\left(y_{1}(1)-4\right)+q_{1}\right)\left(z_{1}(1)-y_{1}(1)\right)+ \\
+\left(2000\left(y_{2}(1)-3\right)+q_{2}\right)\left(z_{2}(1)-y_{2}(1)\right)+q_{3}\left(z_{3}(1)-y_{3}(1)\right) .
\end{gathered}
$$

For admissible controls $\sigma \in \Omega, \sigma^{I} \in \Omega$ denote $p\left(\tau, \sigma^{I}, \sigma\right), \tau \in[0,1]$ - solution of the modified conjugate system with $y(\tau)=y\left(\tau, \sigma^{I}\right), z(\tau)=y(\tau, \sigma), v(\tau)=v^{I}(\tau), \omega=\omega^{I}$.

System conditions for improving permissible $\sigma^{I} \in \Omega$ at $\alpha>0$ in the form of the fixedpoint problem (9) - (13) for the control operator uniquely determined by the method indicated above, in this example takes the form:

$$
\begin{aligned}
& v(\tau)=P_{U}\left(v^{I}(\tau)+\alpha \omega^{I} p_{3}\left(\tau, \sigma^{I}, \sigma\right)\right), \quad \tau \in[0,1] \\
& \omega=P_{W}\left(\omega^{I}+\alpha\left(-1+\int_{T}\left(p_{1}\left(\tau, \sigma^{I}, \sigma\right) \cos y_{3}(\tau, \sigma)+\right.\right.\right. \\
& \left.\left.\left.+p_{2}\left(\tau, \sigma^{I}, \sigma\right) \sin y_{3}(\tau, \sigma)+p_{3}\left(\tau, \sigma^{I}, \sigma\right) v(\tau)\right) d \tau\right)\right) .
\end{aligned}
$$


To calculate the obtained fixed point problem, the following iterative process for $k \geq 0$ with given initial approximation $\sigma^{0}=\left(v^{o}, \omega^{o}\right) \in \Omega$ is considered:

$$
\begin{gathered}
v^{k+1}(\tau)=P_{U}\left(v^{I}(\tau)+\alpha \omega^{I} p_{3}^{k}\left(\tau, \sigma^{I}, \sigma^{k}\right)\right), \quad \tau \in[0,1], \\
\omega^{k+1}=P_{W}\left(\omega^{I}+\alpha\left(-1+\int_{T}\left(p_{1}^{k}\left(\tau, \sigma^{I}, \sigma^{k}\right) \cos y_{3}^{k}\left(\tau, \sigma^{k}\right)+\right.\right.\right. \\
\left.\left.\left.+p_{2}^{k}\left(\tau, \sigma^{I}, \sigma^{k}\right) \sin y_{3}^{k}\left(\tau, \sigma^{k}\right)+p_{3}^{k}\left(\tau, \sigma^{I}, \sigma^{k}\right) v^{k}(\tau)\right) d \tau\right)\right) .
\end{gathered}
$$

The numerical solution of the phase and conjugate Cauchy problems was carried out by the fifth-order or sixth-order Runge-Kutta-Werner method using the Fortran PowerStation 4.0 IMSL library. The values of controllable, phase and conjugate variables were memorized at the nodes of fixed uniform grid $T_{h}$ with discretization step $h>0$. In the intervals between neighboring grid $T_{h}$ nodes, the value of the control function was assumed constant and equal to the value in the left node.

If in the process of calculating the fixed point problem the condition for the first improvement of control was fulfilled $\sigma^{I} \in \Omega$ :

$$
\Phi\left(\sigma^{k+1}\right)+\varepsilon_{1}<\Phi\left(\sigma^{I}\right)
$$

where $\varepsilon_{1} \geq 0$ is a given accuracy of control improvement, a new fixed-point problem was constructed to improve the received calculation control, and the iterative algorithm was repeated.

If no improvement in this sense occurs, then the numerical calculation of the fixed point problem was carried out until the condition $\left\|\sigma^{k+1}-\sigma^{k}\right\|_{T_{h}} \leq \varepsilon_{2}$ is satisfied, where $\varepsilon_{2}>0$ is the specified accuracy of the calculation of the fixed point problem. On this, the construction and calculation of successive problems of improving control ended.

For an adequate comparison of the results of calculating the initial problem, the proposed approach of fixed points with the methods of [2] was used to select similar calculation conditions: $h=10^{-2}, \varepsilon_{1}=10^{-7}, \varepsilon_{2}=10^{-10},\left\|\sigma^{k+1}-\sigma^{k}\right\|_{T_{h}}=\max \left\{\mid \omega^{k+1}-\right.$ $\left.\omega^{k}|,| v^{k+1}(t)-v^{k}(t) \mid, t \in T_{h}\right\}$.

Table 1 shows the results of calculating

Table 1

the Zermelo problem under consideration with the proposed fixed-point method (FPM) in comparison with the results of calculations by computational multimethod technologies [2], in the notations used in this paper. Table 1 indicates $\Delta \Phi^{*}=\left|\Phi^{*}-5,110061\right|, \Phi^{*}$ is the calculated value of the functional, $N$ is number of calculated phase and conjugate Cauchy problems.

Table 2 shows the results of the calculation of the FPM algorithm for different values of projection parameter $\alpha>0$ and initial admissible approximation $\sigma^{0}$. At $N \geq 200000$ the calculation of the problem was stopped. 
Table 2

\begin{tabular}{|l|l|l|l|l|}
\hline$\alpha$ & $v^{0}$ & $\omega^{0}$ & $\Delta \Phi^{*}$ & $N$ \\
\hline $10^{-6}$ & 0,5 & 1 & $1,0-3$ & 23055 \\
\hline $10^{-6}$ & 0,5 & 4 & $1,0-3$ & 20239 \\
\hline $10^{-6}$ & 0,5 & 5 & $6,8-4$ & 12271 \\
\hline $10^{-6}$ & 0,5 & 6 & $7,7-1$ & 200000 \\
\hline $10^{-6}$ & 0,5 & 10 & 4,8 & 200000 \\
\hline $10^{-6}$ & 0 & 1 & $1,0-3$ & 23065 \\
\hline $10^{-6}$ & 0 & 4 & $1,0-3$ & 20245 \\
\hline $10^{-6}$ & 0 & 5 & $9,1-4$ & 14339 \\
\hline $10^{-6}$ & 0 & 6 & $7,1-1$ & 200000 \\
\hline $10^{-6}$ & 0 & 10 & Does not converge & \\
\hline $10^{-6}$ & $-0,5$ & 1 & $1,0-3$ & 23091 \\
\hline $10^{-6}$ & $-0,5$ & 4 & $1,0-3$ & 20219 \\
\hline $10^{-6}$ & $-0,5$ & 5 & $7,9-4$ & 14007 \\
\hline $10^{-6}$ & $-0,5$ & 6 & $7,4-1$ & 200000 \\
\hline $10^{-6}$ & $-0,5$ & 10 & 4,8 & 200000 \\
\hline $10^{-7}$ & 0,5 & 5 & $1,3-3$ & 90695 \\
\hline $10^{-5}$ & 0,5 & 5 & Does not converge & \\
\hline
\end{tabular}

These and other calculations showed that for $\alpha>10^{-5}$ the algorithm does not converge, but as the value of $\alpha$ decreases $10^{-6}$ the convergence of the algorithm slows down significantly and the number of computational problems of the Cauchy increases accordingly. The computational efficiency of the algorithm, estimated by the number of computational problems of Cauchy, also depends significantly on the choice of the initial approximation. As initial $\omega^{0}$ approaches from the left to the conditionally optimal value of $\hat{\Phi}=5,110061$, the improvement in the convergence of the algorithm with respect to computational efficiency and accuracy is observed. When $\omega^{0}$ is removed to the right of the conditionally optimal value of $\hat{\Phi}$, the rate of convergence decays drastically.

\section{The Problem of Optimal Orientation of an Aircraft in Space}

We consider the problem of optimal speed for the orientation of an aircraft in space, which in [2] is reduced by the method of penalty functionals to the following problem:

$$
\begin{aligned}
& \begin{cases}\dot{x}_{1}(t)=x_{3}(t), & x_{1}(0)=10, \\
\dot{x}_{2}(t)=x_{4}(t), & x_{2}(0)=0, \\
\dot{x}_{3}(t)=-x_{4}(t)+u_{1}(t) \sin u_{2}(t), & x_{3}(0)=0, \\
\dot{x}_{4}(t)=x_{3}(t)+u_{1}(t) \cos u_{2}(t), & x_{4}(0)=0,\end{cases} \\
& t \in\left[0, t_{1}\right], \quad 0 \leq u_{1}(t) \leq 1, \quad-\pi \leq u_{2}(t) \leq \pi, u=\left(u_{1}, u_{2}\right), \\
& G\left(u, t_{1}\right)=t_{1}+1000\left(x_{1}^{2}\left(t_{1}\right)+x_{2}^{2}\left(t_{1}\right)+x_{3}^{2}\left(t_{1}\right)+x_{4}^{2}\left(t_{1}\right)\right) \rightarrow \min .
\end{aligned}
$$

In the problem, as in the first example, it can be assumed that $t_{1} \leq \bar{t}, \bar{t}>0$. Time replacement by rule

$$
\begin{gathered}
t(\tau)=t_{0}+\left(t_{1}-t_{0}\right) \tau=\omega \tau, \quad t_{0}=0, \quad \tau \in[0,1], \quad \omega=t_{1} \geq 0, \\
v(\tau)=u(t(\tau)), \quad y(\tau)=x(t(\tau)), \quad \sigma=(v, w),
\end{gathered}
$$


this problem reduces to a nonlinear control problem with a fixed time and mixed control functions and parameters

$$
\begin{gathered}
\begin{cases}\dot{y}_{1}(\tau)=\omega y_{3}(\tau), & y_{1}(0)=10, \\
\dot{y}_{2}(\tau)=\omega y_{4}(\tau)(t), & y_{2}(0)=0, \\
\dot{y}_{3}(\tau)=-\omega y_{4}(\tau)+\omega v_{1}(\tau) \sin v_{2}(\tau), & y_{3}(0)=0, \\
\dot{y}_{4}(\tau)=\omega y_{3}(\tau)+\omega v_{1}(\tau) \cos v_{2}(\tau), & y_{4}(0)=0,\end{cases} \\
\Phi(\sigma)=\omega+1000\left(y_{1}^{2}(1)+y_{2}^{2}(1)+y_{3}^{2}(1)+y_{4}^{2}(1)\right) \rightarrow \min ,
\end{gathered}
$$

The Pontryagin function in the transformed problem looks like this:

$$
H(p, y, v, \omega, \tau)=\omega\left(\left(p_{1}+p_{4}\right) y_{3}+\left(p_{2}-p_{3}\right) y_{4}+\left(p_{3} \sin v_{2}+p_{4} \cos v_{2}\right) v_{1}\right) .
$$

In view of the linearity of the Pontryagin function with respect to variable $y(\tau)$, the modified differential-algebraic conjugate system in accordance with (4) - (7) takes the form:

$$
\begin{cases}\dot{p}_{1}(\tau)=0, & p_{1}(1)=-2000 y_{1}(1)-q_{1}, \\ \dot{p}_{2}(\tau)=0, & p_{2}(1)=-2000 y_{2}(1)-q_{2}, \\ \dot{p}_{3}(\tau)=-\omega\left(p_{1}(\tau)+p_{4}(\tau)\right), & p_{3}(1)=-2000 y_{3}(1)-q_{3}, \\ \dot{p}_{4}(\tau)=-\omega\left(p_{2}(\tau)-p_{3}(\tau)\right), & p_{4}(1)=-2000 y_{4}(1)-q_{4},\end{cases}
$$

in which the value of $q=\left(q_{1}, q_{2}, q_{3}, q_{4}\right)$ is determined from the equation:

$$
\begin{aligned}
& 1000\left(z_{1}^{2}(1)-y_{1}^{2}(1)+z_{2}^{2}(1)-y_{2}^{2}(1)+z_{3}^{2}(1)-y_{3}^{2}(1)+z_{4}^{2}(1)-y_{4}^{2}(1)\right)= \\
& =\left(2000 y_{1}(1)+q_{1}\right)\left(z_{1}(1)-y_{1}(1)\right)+\left(2000 y_{2}(1)+q_{2}\right)\left(z_{2}(1)-y_{2}(1)\right)+ \\
& +\left(2000 y_{3}(1)+q_{3}\right)\left(z_{3}(1)-y_{3}(1)\right)+\left(2000 y_{4}(1)+q_{4}\right)\left(z_{4}(1)-y_{4}(1)\right) .
\end{aligned}
$$

For admissible controls $\sigma \in \Omega, \sigma^{I} \in \Omega$ we denote $p\left(\tau, \sigma^{I}, \sigma\right), \tau \in[0,1]$ - the solution of the modified conjugate system for $y(\tau)=y\left(\tau, \sigma^{I}\right), z(\tau)=y(\tau, \sigma), v(\tau)=v^{I}(\tau), \omega=\omega^{I}$.

The system of conditions for improving admissible $\sigma^{I} \in \Omega$ for $\alpha>0$ in the form of the fixed point problem according to (9) - (13) takes the form:

$$
\begin{gathered}
\left(v_{1}(\tau), v_{2}(\tau)\right)= \\
=P_{U}\left(v_{1}^{I}(\tau)+\alpha\left(\omega^{I}\left(p_{3}\left(\tau, \sigma^{I}, \sigma\right) \sin v_{2}^{I}(\tau)+p_{4}\left(\tau, \sigma^{I}, \sigma\right) \cos v_{2}^{I}(\tau)\right)+s_{1}(\tau)\right),\right. \\
\left.v_{2}^{I}(\tau)+\alpha\left(\omega^{I} v_{1}^{I}(\tau)\left(p_{3}\left(\tau, \sigma^{I}, \sigma\right) \cos v_{2}^{I}(\tau)-p_{4}\left(\tau, \sigma^{I}, \sigma\right) \sin v_{2}^{I}(\tau)\right)+s_{2}(\tau)\right)\right), \tau \in[0,1] \\
\omega=P_{W}\left(\omega^{I}+\alpha\left(-1+\int_{T}\left(\left(p_{1}\left(\tau, \sigma^{I}, \sigma\right)+p_{4}\left(\tau, \sigma^{I}, \sigma\right)\right) y_{3}(\tau, \sigma)+\left(p_{2}\left(\tau, \sigma^{I}, \sigma\right)-\right.\right.\right.\right. \\
\left.\left.\left.\left.-p_{3}\left(\tau, \sigma^{I}, \sigma\right)\right) y_{4}(\tau, \sigma)++v_{1}(\tau)\left(p_{3}\left(\tau, \sigma^{I}, \sigma\right) \sin v_{2}(\tau)+p_{4}\left(\tau, \sigma^{I}, \sigma\right) \cos v_{2}(\tau)\right)\right) d \tau\right)\right),
\end{gathered}
$$

in which value of $s(\tau)=\left(s_{1}(\tau), s_{2}(\tau)\right)$ is determined from the algebraic equation:

$\omega^{I} v_{1}(\tau)\left(p_{3}\left(\tau, \sigma^{I}, \sigma\right) \sin v_{2}(\tau)+p_{4}\left(\tau, \sigma^{I}, \sigma\right) \cos v_{2}(\tau)\right)-\omega^{I} v_{1}^{I}(\tau)\left(p_{3}\left(\tau, \sigma^{I}, \sigma\right) \sin v_{2}^{I}(\tau)+\right.$ $\left.+p_{4}\left(\tau, \sigma^{I}, \sigma\right) \cos v_{2}^{I}(\tau)\right)=\left(\omega^{I}\left(p_{3}\left(\tau, \sigma^{I}, \sigma\right) \sin v_{2}^{I}(\tau)+p_{4}\left(\tau, \sigma^{I}, \sigma\right) \cos v_{2}^{I}(\tau)\right)+s_{1}(\tau)\right)\left(v_{1}(\tau)-\right.$ $\left.-v_{1}^{I}(\tau)\right)+\left(\omega^{I} v_{1}^{I}(\tau)\left(p_{3}\left(\tau, \sigma^{I}, \sigma\right) \cos v_{2}^{I}(\tau)-p_{4}\left(\tau, \sigma^{I}, \sigma\right) \sin v_{2}^{I}(\tau)\right)+s_{2}(\tau)\right)\left(v_{2}(\tau)-v_{2}^{I}(\tau)\right)$. 
We choose the following method of unambiguous resolution of $s(\tau)=\left(s_{1}(\tau), s_{2}(\tau)\right)$ from the algebraic equation:

1) if $v_{1}(\tau) \neq v_{1}^{I}(\tau)$, then

$$
\begin{gathered}
s_{2}(\tau)=0 \\
s_{1}(\tau)=\frac{\omega^{I} v_{1}(\tau)\left(p_{3}\left(\tau, \sigma^{I}, \sigma\right) \sin v_{2}(\tau)+p_{4}\left(\tau, \sigma^{I}, \sigma\right) \cos v_{2}(\tau)\right)}{v_{1}(\tau)-v_{1}^{I}(\tau)}- \\
-\frac{\omega^{I} v_{1}^{I}(\tau)\left(p_{3}\left(\tau, \sigma^{I}, \sigma\right) \sin v_{2}^{I}(\tau)+p_{4}\left(\tau, \sigma^{I}, \sigma\right) \cos v_{2}^{I}(\tau)\right)}{v_{1}(\tau)-v_{1}^{I}(\tau)}- \\
-\frac{\omega^{I} v_{1}^{I}(\tau)\left(p_{3}\left(\tau, \sigma^{I}, \sigma\right) \cos v_{2}^{I}(\tau)-p_{4}\left(\tau, \sigma^{I}, \sigma\right) \sin v_{2}^{I}(\tau)\right)\left(v_{2}(\tau)-v_{2}^{I}(\tau)\right)}{v_{1}(\tau)-v_{1}^{I}(\tau)}- \\
-\omega^{I}\left(p_{3}\left(\tau, \sigma^{I}, \sigma\right) \sin v_{2}^{I}(\tau)+p_{4}\left(\tau, \sigma^{I}, \sigma\right) \cos v_{2}^{I}(\tau)\right) ;
\end{gathered}
$$

2) if $v_{1}(\tau)=v_{1}^{I}(\tau)$, then

2.1) if $v_{2}(\tau) \neq v_{2}^{I}(\tau)$, then

$$
\begin{gathered}
s_{1}(\tau)=0 \\
s_{2}(\tau)=\frac{\omega^{I} v_{1}(\tau)\left(p_{3}\left(\tau, \sigma^{I}, \sigma\right) \sin v_{2}(\tau)+p_{4}\left(\tau, \sigma^{I}, \sigma\right) \cos v_{2}(\tau)\right)}{v_{2}(\tau)-v_{2}^{I}(\tau)}- \\
-\frac{\omega^{I} v_{1}^{I}(\tau)\left(p_{3}\left(\tau, \sigma^{I}, \sigma\right) \sin v_{2}^{I}(\tau)+p_{4}\left(\tau, \sigma^{I}, \sigma\right) \cos v_{2}^{I}(\tau)\right)}{v_{2}(\tau)-v_{2}^{I}(\tau)}- \\
-\omega^{I} v_{1}^{I}(\tau)\left(p_{3}\left(\tau, \sigma^{I}, \sigma\right) \cos v_{2}^{I}(\tau)-p_{4}\left(\tau, \sigma^{I}, \sigma\right) \sin v_{2}^{I}(\tau)\right)
\end{gathered}
$$

2.2) if $v_{2}(\tau)=v_{2}^{I}(\tau)$, then

$$
s_{1}(\tau)=0, s_{2}(\tau)=0 .
$$

To solve the above fixed point problem, similarly to the previous example, we used an explicit iterative algorithm of simple iterations with the same computational features of the implementation:

$$
\begin{aligned}
& \left(v_{1}^{k+1}(\tau), v_{2}^{k+1}(\tau)\right)= \\
& =P_{U}\left(v_{1}^{I}(\tau)+\alpha\left(\omega^{I}\left(p_{3}\left(\tau, \sigma^{I}, \sigma^{k}\right) \sin v_{2}^{I}(\tau)+p_{4}\left(\tau, \sigma^{I}, \sigma^{k}\right) \cos v_{2}^{I}(\tau)\right)+s_{1}^{k}(\tau)\right),\right. \\
& \left.v_{2}^{I}(\tau)+\alpha\left(\omega^{I} v_{1}^{I}(\tau)\left(p_{3}\left(\tau, \sigma^{I}, \sigma^{k}\right) \cos v_{2}^{I}(\tau)-p_{4}\left(\tau, \sigma^{I}, \sigma^{k}\right) \sin v_{2}^{I}(\tau)\right)+s_{2}^{k}(\tau)\right)\right), \tau \in[0,1], \\
& \omega^{k+1}=P_{W}\left(\omega^{I}+\alpha\left(-1+\int_{T}\left(\left(p_{1}\left(\tau, \sigma^{I}, \sigma^{k}\right)+p_{4}\left(\tau, \sigma^{I}, \sigma^{k}\right)\right) y_{3}\left(\tau, \sigma^{k}\right)+\right.\right.\right. \\
& +\left(p_{2}\left(\tau, \sigma^{I}, \sigma^{k}\right)-p_{3}\left(\tau, \sigma^{I}, \sigma^{k}\right)\right) y_{4}\left(\tau, \sigma^{k}\right)+ \\
& \left.\left.\left.+v_{1}^{k}(\tau)\left(p_{3}\left(\tau, \sigma^{I}, \sigma^{k}\right) \sin v_{2}^{k}(\tau)+p_{4}\left(\tau, \sigma^{I}, \sigma^{k}\right) \cos v_{2}^{k}(\tau)\right)\right) d \tau\right)\right),
\end{aligned}
$$

in which value of $s^{k}(\tau)=\left(s_{1}^{k}(\tau), s_{2}^{k}(\tau)\right)$ is determined from the corresponding algebraic equation analogous to the single-valued rule for quantity $s(\tau)$. The operation of projection $P_{U}(z)$ on set $U=\left\{u=\left(u_{1}, u_{2}\right): 0 \leq u_{1} \leq 1, \quad-\pi \leq u_{2} \leq \pi\right\}$ is realized analytically in the form of a simple conditional formula. 
Table 3 shows the data for calculating Table 3 the model problem developed in [2] by multimethod iterative technologies for which the initial initial approximations are not given in [2] for a complete adequate comparison of the approaches considered with the proposed fixed point algorithm. Table 3 uses notation $\Delta \Phi^{*}=$ $\left|\Phi^{*}-10,285456\right|, \Phi^{*}$ is the calculated value of the functional, $N$ is a number of calculated phase and conjugate Cauchy problems.

For comparison, Table 4 shows the results of calculating the problem under consideration by the fixed point method used for $\alpha=10^{-6}$ and various initial approximations. At $N \geq 400000$ the calculation of the problem was stopped.

Table 4

\begin{tabular}{|l|l|l|l|l|}
\hline$v_{0}^{1}$ & $v_{0}^{2}$ & $\omega^{0}$ & $\Delta \Phi^{*}$ & $N$ \\
\hline 1 & 1 & 1 & Does not converge & - \\
\hline 1 & 1 & 5 & $1,4-1$ & 12181 \\
\hline 1 & 1 & 10 & $3,9-3$ & 11805 \\
\hline 1 & 1 & 11 & $7,0-1$ & 125925 \\
\hline 1 & 1 & 15 & 4,7 & 137377 \\
\hline 0,5 & -1 & 1 & $1,4-1$ & 12713 \\
\hline 0,5 & -1 & 5 & $1,4-1$ & 12201 \\
\hline 0,5 & -1 & 10 & $3,9-3$ & 11583 \\
\hline 0,5 & -1 & 11 & $6,7-1$ & 206623 \\
\hline 0,5 & -1 & 15 & 4,5 & 400000 \\
\hline 0,5 & 1 & 1 & Does not converge & - \\
\hline 0,5 & 1 & 5 & $1,4-1$ & 12207 \\
\hline 0,5 & 1 & 10 & $5,1-3$ & 11117 \\
\hline 0,5 & 1 & 11 & $7,0-1$ & 177871 \\
\hline 0,5 & 1 & 15 & 4,5 & 400000 \\
\hline
\end{tabular}

The analysis of these and other calculations performed by the proposed fixed point algorithm demonstrates similarities to the first model example of the dependence of the convergence of the algorithm on the projection parameter $\alpha>0$ and the initial approximations.

\section{Conclusion}

The computational experiments carried out on model speed problems demonstrate the computational and qualitative efficiency of the proposed fixed point approach, acceptable for practice, in comparison with known methods [2]. The developed nonlocal approach to the search for approximate-optimal solutions has a rather wide range of convergence in the initial approximation and is characterized by the convenience and simplicity of the experimental adjustment of the scalar projection parameter that regulates the quality and 
speed of convergence of the iterative process under consideration. Approximately-optimal solutions of optimal speed problems obtained with the help of the proposed approach can be considered as acceptable initial approximations for further iterative refinement by other methods. These features of the proposed approach are important factors for increasing the efficiency and future development of nonlocal methods for solving optimal speed problems.

Acknowledgements. The work was carried out with the financial support of the Ministry of Education and Science of the Russian Federation, project 1.5049.2017/BP; Russian Foundation for Basic Research, project 18-41-030005.

\section{References}

1. Tyatyushkin A.I. Mnogometodnaya tehnologiya optimizacii upravljaemyh sistem [MultiMethod Optimization of Controllable Systems]. Novosibirsk, Nauka, 2006. (in Russian)

2. Gornov A.Yu. Vychislitel'nye tehnologii resheniya zadach optimal'nogo upravleniya [Computational Technologies for Solving Optimal Control Problems]. Novosibirsk, Nauka, 2009. (in Russian)

3. Srochko V.A. Iteracionnye metody resheniya zadach optimal'nogo upravleniya [Iterative Methods for Solving Optimal Control Problems]. Moscow, Fizmatlit, 2000. (in Russian)

4. Buldaev A.S., Khishektueva I.-Kh. The Fixed Point Method in Parametric Optimization Problems for Systems. Automation and Remote Control, 2013, vol. 74, no. 12, pp. 1927-1934.

5. Buldaev A.S., Daneev A.V. New Approaches to Optimization of Parameters of Dynamic Systems on the Basis of Problems about Fixed Points. Far East Journal of Mathematical Sciences, 2016, vol. 99, no. 3, pp. 439-454.

6. Buldaev A.S., Khishketueva I.-Kh. Fixed Point Methods in Problems of Optimization of Nonlinear Systems by Control Functions and Parameters. The Bulletin of Irkutsk State University, Series: Mathematics, 2017, vol. 19, pp. 89-104. (in Russian) DOI: 10.26516/19977670.2017.19.89

7. Buldaev A.S. Methods of Fixed Points on the Basis of Design Operations in Optimization Problems of Control Functions and Parameters of Dynamical Systems. The Buryat State University Bulletin, Series: Mathematics, Informatics, 2017, vol. 1, pp. 38-54. (in Russian) DOI: $10.18101 / 2304-5728-2017-1-38-54$

Received August 14, 2018

УДК 517.977

DOI: $10.14529 / \mathrm{mmp} 180404$

\section{ОБ ОДНОМ ПОДХОДЕ К ЧИСЛЕННОМУ РЕШЕНИЮ НЕЛИНЕЙНЫХ ЗАДАЧ ОПТИМАЛЬНОГО БЫСТРОДЕЙСТВИЯ}

\section{А.С. Булдаев ${ }^{1}$, И.Д. Бурлаков ${ }^{1}$}

${ }^{1}$ Бурятский государственный университет, г. Улан-Удэ, Российская Федерация

Задачи оптимального быстродействия относятся к важнейшим задачам теории управляемых систем. В качественной теории нелинейных задач быстродействия одним из основных результатов является принцип максимума Понтрягина. Для численного решения нелинейных задач быстродействия, наряду с методами, основанными на принципе максимума, широко применяют способы сведения к вспомогательным задачам 
оптимального управления с помощью процедур линеаризации, параметризации, дискретизации и других приемов. Трудоемкость численных методов определяется количеством итераций для нахождения решения задачи быстродействия с заданной точностью. Универсальной вычислительной процедуры, являющейся эффективной для расчета разнообразных задач быстродействия, в настоящее время не существует. Поэтому актуальным является разработка специальных подходов, позволяющих уменьшать объем вычислений и сокращать число итераций. В работе предлагается новый подход, основывающийся на сведении нелинейной задачи оптимального быстродействия к вспомогательной задаче оптимизации со смешанными управляющими функциями и параметрами. Для поиска решения возникающей вспомогательной задачи используются специальная разработанная форма условий нелокального улучшения допустимого управления в виде задачи о неподвижной точке оператора управления и конструируемый итерационный алгоритм последовательного улучшения допустимых управлений. Проводится апробация и сравнительный анализ вычислительной эффективности предлагаемого подхода неподвижных точек на известных модельных задачах оптимального быстродействия.

Ключевые слова: задача оптимального быстродействия; условия улучшения управления; задача о неподвижной точке.

\section{Литература}

1. Тятюшкин, А.И. Многометодная технология оптимизации управляемых систем / А.И. Тятюшкин. - Новосибирск: Наука, 2006.

2. Горнов, А.Ю. Вычислительные технологии решения задач оптимального управления / А.Ю. Горнов. - Новосибирск: Наука, 2009.

3. Срочко, В.А. Итерационные методы решения задач оптимального управления / В.А. Срочко. - М.: Физматлит, 2000.

4. Buldaev, A.S. The Fixed Point Method in Parametric Optimization Problems for Systems / A.S. Buldaev, I.-Kh. Khishektueva // Automation and Remote Control. - 2013. - V. 74, № 12. - P. 1927-1934.

5. Buldaev, A.S. New Approaches to Optimization of Parameters of Dynamic Systems on the Basis of Problems about Fixed Points / A.S. Buldaev, A.V. Daneev // Far East Journal of Mathematical Sciences. - 2016. - V. 99, № 3. - P. 439-454.

6. Булдаев, А.С. Методы неподвижных точек в задачах оптимизации нелинейных систем по управляющим функциям и параметрам / А.С. Булдаев, И.-Х.Д. Хишектуева // Известия Иркутского государственного университета. Серия: Математика. - 2017. - Т. 19. C. $89-104$.

7. Булдаев, А.С. Методы неподвижных точек на основе операций проектирования в задачах оптимизации управляющих функций и параметров динамических систем / А.С. Булдаев // Вестник Бурятского государственного университета. Серия: Математика, информатика. - 2017. - № 1. - С. 38-54.

Александр Сергеевич Булдаев, доктор физико-математических наук, профессор, кафедра прикладной математики, Бурятский государственный университет (г. УланУдэ, Российская Федерация), buldaev@mail.ru.

Иван Дмитриевич Бурлаков, ассистент, кафедра прикладной математики, Бурятский государственный университет (г. Улан-Удэ, Российская Федерация), ivan.burlakov.91@mail.ru.

Поступила в редакиию 14 августа 2018 г. 